\title{
Integral test of natural tungsten for CENDL-3.1
}

Liu Ping ${ }^{\mathrm{a}}$

China Nuclear Data Center, China Institute of Atomic Energy, P.O. Box 275(41), Beijing 102413, P.R. China

\begin{abstract}
Integral experiment testing was carried out for neutron leakage spectra experiment from 40-cm tungsten pile and criticality experiment with tungsten reflector using the continuous-energy Monte Carlo code MCNP and the different natural tungsten data generated from CENDL-3.1, FENDL-2.0, ENDF/B-VII and JENDL-3.2. From the benchmark test results, we can say that neutron leakage spectrum obtained with CENDL-3.1 shows good agreement with experimental measurement below $5 \mathrm{MeV}$, but the leakage spectra at $5 \sim 13 \mathrm{MeV}$ were underestimated with CENDL-3.1, JENDL-3.2 and ENDF/B-VII, especially the CENDL-3.1, but FENDL-2.0 gives better result in this energy range. For criticality experiment, CENDL-3.1 underestimates criticality by $514 \mathrm{pcm}$. Further improvement need to be made for tungsten in CENDL-3.1.
\end{abstract}

\section{Introduction}

Tungsten alloy is a very important structure material for nuclear reactor. Though there is small absorption, the precision of the evaluation for tungsten still has fairly effect on the calculation of $\mathrm{k}_{\mathrm{eff}}$ values, because large quantity of tungsten alloy is utilized. Therefore, benchmark testing for tungsten is very important. The latest natural tungsten data of CENDL3.1 (China Evaluated Nuclear Data Library) were released in 2005. It's necessary to do the benchmark testing for natural tungsten data of CENDL-3.1. Neutron leakage spectrum experiment from tungsten pile and criticality experiment with tungsten reflector are considered in the test. The continuousenergy Monte Carlo code MCNP [1] was used for neutron transport calculation. The ACE format files for MCNP were generated using NJOY 97 [2]. The calculated results with CENDL-3.1, FENDL-2.0, ENDF/B-VII and JENDL-3.2 are compared with the experimental results.

\section{Comparison of tungsten evaluation}

In this paper we present the review and analysis of $(n, 2 n)$, total inelastic cross section and discrete inelastic scattering cross section for tungsten. The comparisons for total inelastic cross-section of natural tungsten from CENDL-3.1, ENDF/BVII and JENDL-3.2 are given in figure 1. It can be seen that the cross section of CENDL-3.1 is lower, and the ones from EMDF/B-VII and JENDL-3.2 are close to the experimental ones. Figure 2 shows the comparison of (n,2n) cross sections. It can be seen that tungsten of CENDL-3.1 is similar to the one of JENDL-3.2, and the (n,2n) cross section of ENDF/B-VII is close to the experimental one. Figure 3 shows the comparison of discrete inelastic scattering cross section. The cross section of CENDL-3.1 is lower compared with those of ENDF/B-VII and JENDL-3.2.

\footnotetext{
${ }^{a}$ e-mail: ping@iris.ciae.ac.cn
}

\section{Benchmark testing and analyses}

\subsection{Neutron leakage spectra experiment}

Neutron leakage spectra experiment from $40-\mathrm{cm}$ tungsten pile was considered in this testing. The experiment was performed with the time-of-flight (TOF) technique using the intense $14 \mathrm{MeV}$ neutron source facility OKTAVIAN $[3,4]$ at Osaka University. A tritium neutron-producing target was placed at the center of the pile. In benchmark calculation, source angular distribution was supposed to be isotropic. Neutron source spectrum supplied by the experimentalists was utilized in MCNP calculation. Result obtained from CENDL-3.1, FENDL-2.0, ENDF/B-VII and JENDL-3.2 is compared with experimental measurement in figure 4.

In general, neutron leakage spectrum obtained with CENDL-3.1 shows good agreement with experimental measurement below $5 \mathrm{MeV}$. The leakage spectra at $5 \sim 13 \mathrm{MeV}$ were underestimated with CENDL-3.1, JENDL-3.2 and ENDF/B-VII, especially the CENDL-3.1, but FENDL-2.0 gives better result in this range. This may be due to the underestimations of total inelastic cross section and discrete inelastic scattering cross section of tungsten from CENDL3.1. Improvements needs to be made for CENDL-3.1.

\subsection{Criticality experiment with tungsten reflector}

Critical assembly ${ }^{233}$ U-MET-FAST-004 [5] with tungsten reflector were considered in the present study.

\subsubsection{Description of the model}

The assembly ${ }^{233} \mathrm{U}$-MET-FAST-004 is a simple highly enriched ${ }^{233} \mathrm{U}$ sphere reflected by tungsten. The model is an idealized configuration derived by the experiments. Tables 1 and 2 show brief benchmark composition. 


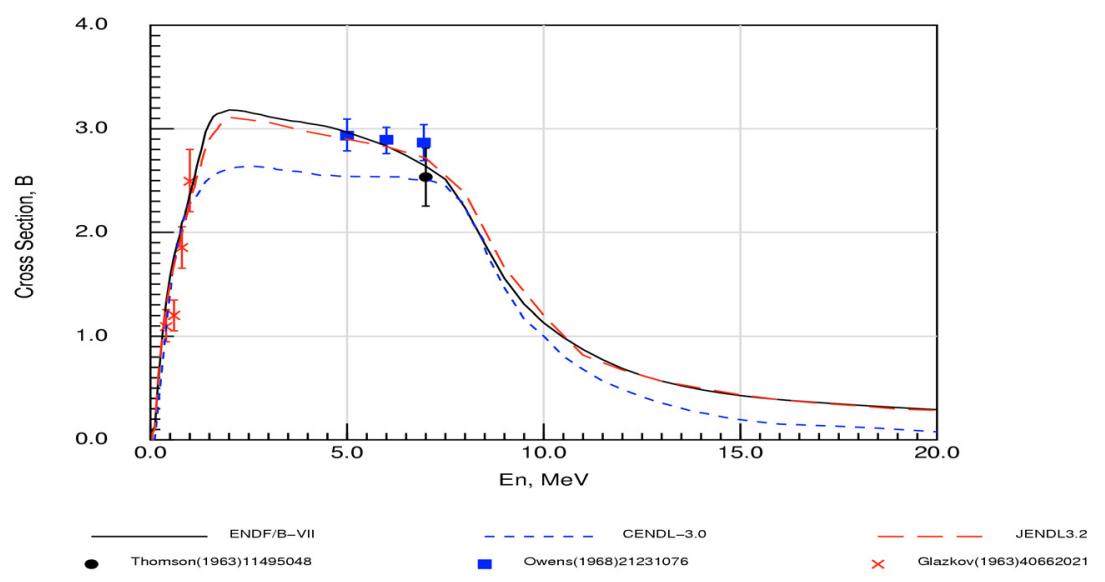

Fig. 1. Comparison of total inelastic cross sections for natural W.

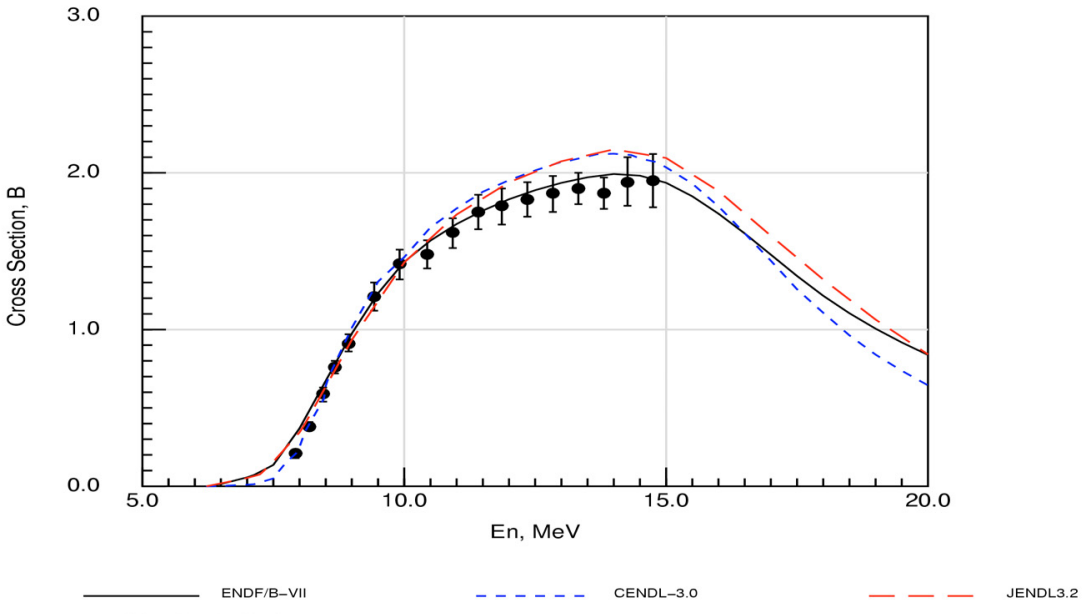

- Frehaut(1980)20416017

Fig. 2. Comparison of total $(\mathrm{n}, 2 \mathrm{n})$ cross sections for natural $\mathrm{W}$.

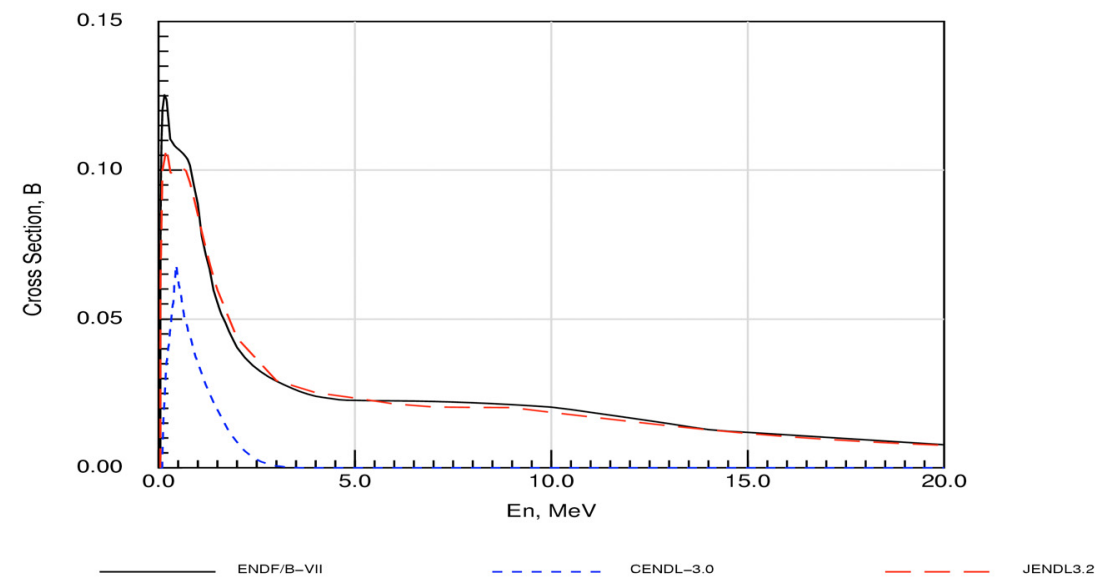

Fig. 3. Comparison of discrete inelastic scattering cross sections for natural W (MT = 51). 


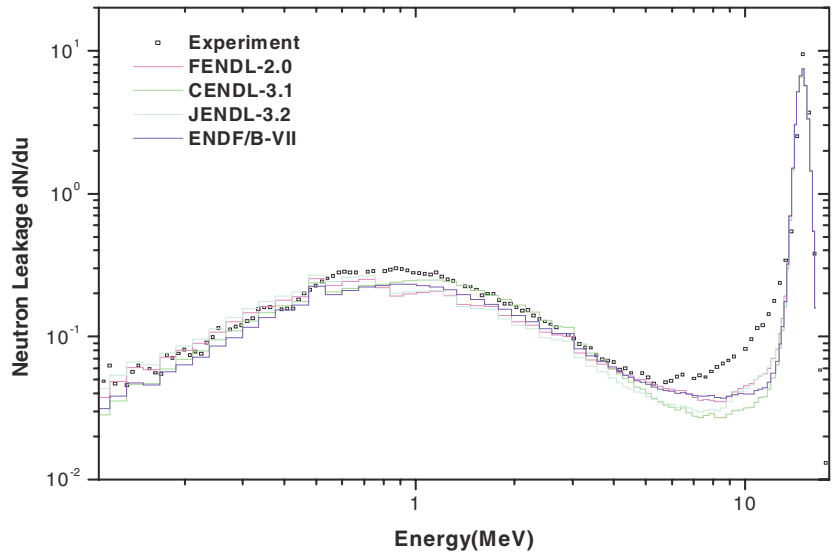

Fig. 4. Neutron leakage spectrum from $\mathrm{W}$ pile of $40 \mathrm{~cm}$.

Table 1. ${ }^{233} \mathrm{U}$ core composition.

\begin{tabular}{|c|c|}
\hline Isotope & Wt. \% \\
\hline${ }^{233} \mathrm{U}$ & 98.2 \\
\hline${ }^{234} \mathrm{U}$ & 1.1 \\
\hline${ }^{238} \mathrm{U}$ & 0.7 \\
\hline
\end{tabular}

Table 2. Composition of the tungsten reflector.

\begin{tabular}{|c|c|}
\hline Isotope & Wt. \% \\
\hline Tungsten & 91.3 \\
\hline Nickel & 5.5 \\
\hline Copper & 2.5 \\
\hline Zirconium & 0.7 \\
\hline
\end{tabular}

\subsubsection{MCNP calculation}

The benchmark calculations were performed by using the continuous-energy Monte Carlo code MCNP with four different nuclear data libraries: CENDL-3.1, JENDL-3.2, FENDL2.0 and $\mathrm{ENDF} / \mathrm{B}-\mathrm{VII}$.

In each MCNP calculation, the first 100 cycles were skipped, followed by 1,000 active cycles, each with 10,000 particles per cycle. Statistic error of $\mathrm{k}_{\mathrm{eff}}$ values are within the range from 0.00019 to 0.00021 .

\subsubsection{Results and discussions}

Table 3 shows the $(C / E-1)$ values of $k_{\text {eff }}$ for assembly of highly enriched ${ }^{233} \mathrm{U}$ sphere reflected by tungsten.
Table 3. Comparison of $\mathrm{k}_{\mathrm{eff}}$ for U233-MET-FAST-004.

\begin{tabular}{||l|c|c||}
\hline \hline & $\mathrm{k}_{\mathrm{eff}}$ & $(\mathrm{C} / \mathrm{E}-1)(\mathrm{pcm})$ \\
\hline Experiment & $1.0000 \pm 0.0007$ & - \\
\hline CENDL-3.1 & $0.99486 \pm 0.00019$ & -514 \\
\hline JENDL-3.2 & $1.00344 \pm 0.00020$ & -153 \\
\hline FENDL-2.0 & $0.99298 \pm 0.00019$ & -702 \\
\hline ENDF/B-VII & $0.99847 \pm 0.00020$ & 344 \\
\hline \hline
\end{tabular}

From the table 3, the following is observed:

- The calculated $\mathrm{k}_{\mathrm{eff}}$ values are increasing in the order of the FENDL-2.0, CENDL-3.1, ENDF/B-VII and JENDL3.2 results.

- The CENDL-3.1 underestimates criticality by $514 \mathrm{pcm}$, may be the underestimated discrete inelastic scattering leads to higher average energy of neutron and smaller $\mathrm{k}_{\mathrm{eff}}$. Figures 1-3 show the comparisons of inelastic cross section and $(n, 2 n)$ cross section from different nuclear data libraries.

- JENDL-3.2 gives the most accurate result.

\section{Conclusion}

Underestimation of total inelastic cross-section and discretely inelastic scattering cross-section should be modified for tungsten in the future revised version of CENDL-3.1, and the $(n, 2 n)$ reaction should also be improved for tungsten in CENDL-3.1.

\section{References}

1. J.F. Briesmeister, $M C N P^{T M}-A$ General Monte Carlo N-Particle Transport Code, LA-13709-M, Los Alamos National Laboratory Report (2000).

2. R.E. MacFarlane, D.W. Muir, The NJOY Nuclear Data Processing System, LA-12740-M, Los Alamos National Laboratory Report (1994).

3. C. Ichihara, K. Kobayashi, S.A. Hayashi, I. Kimura, J. Yamamoto, A. Takahashi, Leakage Neutron Spectra from Various Sphere Piles with $14 \mathrm{MeV}$ Neutrons, JAERI-M 94-014 (1994), pp. 63-125.

4. Fusion Evaluated Nuclear Data Library CDROM, IAEA-NDSCD-6.

5. International Hand Book of Evaluated Criticality Safety Benchmark Experiments, NEA/NSC/DOC (95), Nuclear Energy Agency, OECD, Paris (Sept. 2003). 